\title{
Lorentz boost and non-Gaussianity in multi-field DBI-inflation
}

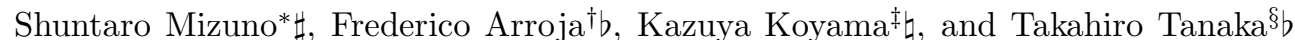 \\ $\sharp S c h o o l$ of Physics and Astronomy, University of Nottingham, University Park, \\ Nottingham NG7 2RD, UK; Research Center for the Early Universe (RESCEU), \\ Graduate School of Science, The University of Tokyo, Tokyo 113-0033, Japan. \\ bYukawa Institute for Theoretical Physics, Kyoto University, Kyoto 606-8502, Japan. \\ $\square$ Institute of Cosmology and Gravitation, University of Portsmouth, Portsmouth PO1 3FX, UK.
}

(Dated: July 6, 2009)

\begin{abstract}
We show that higher-order actions for cosmological perturbations in the multi-field DBI-inflation model are obtained by a Lorentz boost from the rest frame of the brane to the frame where the brane is moving. We confirm that this simple method provides the same third- and fourth- order actions at leading order in slow-roll and in the small sound speed limit as those obtained by the usual ADM formalism. As an application, we compute the leading order connected four-point function of the primordial curvature perturbation coming from the intrinsic fourth-order contact interaction in the multi-field DBI-inflation model. At third order, the interaction Hamiltonian arises purely by the boost from the second-order action in the rest frame of the brane. The boost acts on the adiabatic and entropy modes in the same way thus there exists a symmetry between the adiabatic and entropy modes. But at fourth order this symmetry is broken due to the intrinsic fourth-order action in the rest frame and the difference between the Lagrangian and the interaction Hamiltonian. Therefore, contrary to the three-point function, the momentum dependence of the purely adiabatic component and the components including the entropic contributions are different in the four-point function. This suggests that the trispectrum can distinguish the multi-field DBI-inflation model from the single field DBI-inflation model.
\end{abstract}

\section{INTRODUCTION}

Precise measurements of the cosmic microwave background (CMB) anisotropies such as those obtained by the WMAP satellite [1] provide valuable information on the very early universe. Any theoretical model that attempts to explain the evolution of the universe before the big bang nucleosynthesis will also have to explain the observed CMB anisotropies. Even though these anisotropies are almost Gaussian, a small amount of non-Gaussianity is still allowed by the data [2, 3, 4]. The information contained in this non-Gaussian component will contribute to a huge advance in our understanding of the very early universe. For example, the simplest slow-roll single field inflation models predict that the non-Gaussianity of the fluctuations should be very difficult to be detected [5], even in future experiments such as PLANCK [6]. If we detect large non-Gaussianity, this means that the simplest model of slow-roll single field inflation would be rejected.

Recently, theoretical models which can produce sizeable non-Gaussianity has been extensively studied by many

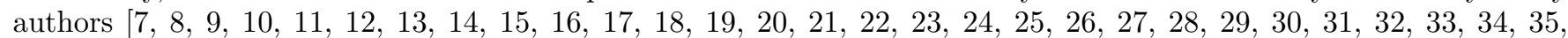
36, 37, 38, 39, 40, 41, 42, 43, 44, 45, 46, 47, 48, 49, 50, 51, 52, 53, 54, 55, 56, 57, 58, 59, 60, 61]. They basically relax one (or more) of the following standard conditions: single field, slow-roll, canonical kinetic term and standard Bunch-Davies vacuum. In the present work, we will consider the case of multiple fields with non-canonical kinetic terms. For example, in $K$-inflation models where the kinetic term of the inflaton field is generic, the sound speed of the perturbations can be much smaller than 1 [62, 63], which leads to large non-Gaussianity.

Among the models, the Dirac-Born-Infeld (DBI) inflation, motivated by string theory, can also realize large nonGaussianity [13, 27, 64, 65, 66, 67]. In this model, the inflaton is identified with the position of a moving D3 brane whose dynamics is described by the DBI action. Again, due to the non-trivial form of the kinetic term, the sound speed can be smaller than 1 and the non-Gaussianity becomes large. However, recently it has been pointed out that DBI-inflation driven by a mobile D3 brane with large non-Gaussianity might contradict the current WMAP data. For current and stringent observational constraints and consequences of DBI-inflation see 668, 69, 70, 71, 72, 73, 74, 75, 76, 77.

One way to avoid this constraint is to consider multi-field DBI models [36]. Since the position of the brane in each compact direction is described by a scalar field, DBI-inflation is naturally a multi-field inflationary model [78]. As

\footnotetext{
* shuntaro.mizuno@nottingham.ac.uk

$\dagger$ arrojaf@yukawa.kyoto-u.ac.jp

$\ddagger$ Kazuya.Koyama@port.ac.uk

$\S$ tanaka@yukawa.kyoto-u.ac.jp
} 
first pointed out by [79], in multi-field inflation models, the curvature perturbation is modified on large scales due to the entropy perturbation. Even though there are some works considering multi-field inflationary models with kinetic terms depending on $X=-G_{I J} \partial_{\mu} \phi^{I} \partial^{\mu} \phi^{J} / 2$, where $\phi^{I}$ are the scalar fields $(I=1,2, \ldots)$ and $G_{I J}$ is the metric in the field space as in the case of K-inflation [45, 80, 81] , the consistent analysis for the entropy modes in the multi-field DBI-inflation model has started only very recently [35, 36, 37, 55, 57].

In [35, 36, 37, 57], the three-point function in the small sound speed limit and at leading order in the slowroll expansion was obtained and it was shown that in addition to the purely adiabatic three-point function, there exists a mixed component $\left\langle Q_{\sigma}\left(\mathbf{k}_{\mathbf{1}}\right) Q_{s}\left(\mathbf{k}_{\mathbf{2}}\right) Q_{s}\left(\mathbf{k}_{\mathbf{3}}\right)\right\rangle$ where $Q_{\sigma}$ and $Q_{s}$ are the adiabatic and the entropy perturbations, respectively. Since the momentum dependence of the three-point function from the adiabatic modes was shown to be identical with the mixed component, the shape of the bispectrum of the curvature perturbations remains the same as in the single-field case, while the amplitude is affected by the entropy perturbation.

The previous works on the non-Gaussianity in the multi-field DBI-inflation model are limited to the bispectrum, with the exception of [55], where the authors compute the leading order trispectrum based on the assumption that it is mainly from some limited terms of the entropy perturbations. It is expected that the cosmic microwave background (CMB) trispectrum also provides strong constraints on early universe models. At the moment, the constraints are rather weak given by $\left|\tau_{N L}\right|<10^{8}$ [82, 83], where $\tau_{N L}$ denotes the size of the trispectrum. However, PLANCK will tighten the constraints significantly reaching $\left|\tau_{N L}\right| \sim 560$ [84]. It is also worth noting that the analysis in the single field DBI-inflation model shows that the trispectrum is enhanced in the small sound speed limit as $\tau_{N L} \sim 1 / c_{s}^{4}$ [28, 85, 86]. As in the bispectrum case, the constraints depend on the shape of the wave vectors' configuration [87]. Therefore, it is important to calculate the shape dependence of the trispectrum in the multi-field DBI-inflation model. For the details of the observations of the CMB trispectrum, see [88, 89, [90].

In this paper, we calculate the four-point function of the primordial curvature perturbation coming from the intrinsic fourth-order contact interaction in the multi-field DBI-inflation model and see whether the momentum dependence of the four-point function is useful to discriminate the multi-field DBI-inflation model or not. In order to obtain the third- and fourth- order actions for cosmological perturbations, we propose a simple and intuitive method based on a Lorentz boost, making use of the special property of the DBI action. This does not only provide the fourth-order action easily, but also explains why the momentum dependence of the three-point function from adiabatic modes is identical with the one from the mixed component.

The structure of this paper is as follows. In section II we describe our model and define perturbations in the flat gauge. We decompose the perturbations into the adiabatic and entropy directions. In section III, after obtaining the fourth-order action at leading order in slow-roll and in the small sound speed limit in terms of the decomposed fields based on the ADM formalism [5, 17, 18, 91], we calculate the fourth-order interaction Hamiltonian. Then, the four-point functions coming from the intrinsic fourth-order contact interaction are derived in section IV] In section V in order to develop understanding of the results, we derive the same fourth-order action based on a Lorentz boost which relates the brane-rest frame and the brane-moving frame. Section VI is devoted to the conclusion.

\section{THE MODEL}

We start with the multi-field DBI-inflation model described by the following action 92

$$
\begin{aligned}
& S=\frac{1}{2} \int d^{4} x \sqrt{-g}\left[R+2 \tilde{P}\left(\tilde{X}, \phi^{I}\right)\right], \\
& \tilde{P}\left(\tilde{X}, \phi^{I}\right)=-\frac{1}{f\left(\phi^{I}\right)}\left(\sqrt{1-2 f\left(\phi^{I}\right) \tilde{X}}-1\right)-V\left(\phi^{I}\right),
\end{aligned}
$$

where we have set $8 \pi G=1, R$ is the Ricci scalar, $\phi^{I}$ are the scalar fields $(I=1,2, \ldots, N), f\left(\phi^{I}\right)$ and $V\left(\phi^{I}\right)$ are functions of the scalar fields determined by string theory configurations and $\tilde{X}$ is defined in terms of the determinant $\mathcal{D} \equiv \operatorname{det}\left(\delta_{\nu}^{\mu}+f G_{I J} \partial^{\mu} \phi^{I} \partial_{\nu} \phi^{J}\right)$ as $\tilde{X}=(1-\mathcal{D}) /(2 f)$. Here $G_{I J}$ is the metric in the field space. We assume that $\tilde{P}$ is a well behaved function of $\phi^{I}$ and $\tilde{X}$. It is also shown that $\tilde{X}$ is related to the kinetic terms of the scalar fields as [36, 37]

$$
\begin{aligned}
\tilde{X} & =G_{I J} X^{I J}-2 f X_{I}^{[I} X_{J}^{J]}+4 f^{2} X_{I}^{[I} X_{J}^{J} X_{K}^{K]}-8 f^{3} X_{I}^{[I} X_{J}^{J} X_{K}^{K} X_{L}^{L]}, \\
X^{I J} & \equiv-\frac{1}{2} g^{\mu \nu} \partial_{\mu} \phi^{I} \partial_{\nu} \phi^{J}, \quad X_{I}^{J}=G_{I K} X^{K J},
\end{aligned}
$$

where the brackets denote antisymmetrization. It is worth noting that even though $\tilde{X}=X\left(=G_{I J} X^{I J}\right)$ in the homogeneous background, this does not hold if we take into account the inhomogeneous components. 
In the background, we are interested in flat, homogeneous and isotropic Friedman-Robertson-Walker universes described by the line element

$$
d s^{2}=-d t^{2}+a^{2}(t) \delta_{i j} d x^{i} d x^{j}
$$

where $a(t)$ is the scale factor. The Friedman equation and the continuity equation read

$$
\begin{gathered}
3 H^{2}=E_{0}, \\
\dot{E}_{0}=-3 H\left(E_{0}+\tilde{P}_{0}\right),
\end{gathered}
$$

where the Hubble rate is $H=\dot{a} / a$, dot denotes derivative with respect to cosmic time $t, E_{0}$ is the total energy of the fields which is given by

$$
E_{0}=2 X_{0}^{I J} \tilde{P}_{0, X^{I J}}-\tilde{P}_{0}
$$

and the subscript 0 denotes that the quantity is evaluated in the background.

For the later convenience, we introduce the following parameter that characterizes the motion of the brane in this background and serves as a Lorentz factor

$$
\gamma\left(\phi_{0}^{I}, X_{0}\right) \equiv \frac{1}{\sqrt{1-v^{2}}}, \quad \text { with } \quad v \equiv \sqrt{2 f_{0} X_{0}},
$$

where we have used the fact that $\tilde{X}_{0}=X_{0}$.

For this model the speed of propagation of the scalar perturbations ("speed of sound"), $c_{s}$, is given by

$$
c_{s}^{2} \equiv\left(\frac{\tilde{P}_{, \tilde{X}}}{\tilde{P}_{, \tilde{X}}+2 \tilde{X} \tilde{P}_{, \tilde{X} \tilde{X}}}\right)_{0} .
$$

It can be shown that $c_{s}$ is the inverse of $\gamma$.

Since we are interested in the inflationary background, we assume the form of $f\left(\phi^{I}\right)$ and $V\left(\phi^{I}\right)$ are chosen so that the inflation is realized at least for 60 e-foldings. In order to characterize this background, we define the slow-variation parameters, analogues of the slow-roll parameters, as

$$
\epsilon=-\frac{\dot{H}}{H^{2}}=\frac{X_{0}}{H^{2} c_{s}}, \quad \eta=\frac{\dot{\epsilon}}{\epsilon H}, \quad \chi=\frac{\dot{c_{s}}}{c_{s} H} .
$$

We should note that these slow-variation parameters are more general than the usual slow-roll parameters and that the smallness of these parameters does not imply that the field in rolling slowly. We assume that the rate of change of the speed of sound is small (as described by $\chi$ ) but $c_{s}$ is otherwise free to change between zero and one.

We shall consider perturbations on this background. We decompose the scalar field $\phi^{I}$ into the background value $\phi_{0}^{I}$ and the perturbation $Q^{I}$ in the flat gauge as,

$$
\phi^{I}(x, t)=\phi_{0}^{I}(t)+Q^{I}(x, t) .
$$

Furthermore, as was done in 93], we decompose the perturbations into instantaneous adiabatic and entropy perturbations, where the adiabatic direction corresponds to the direction of the background fields' evolution while the entropy directions are orthogonal to this. We introduce an orthogonal basis $e_{n}^{I}$, with $n=1,2, \ldots, N$, in the field space so that the orthonormality conditions are given by 35

$$
e_{n}^{I} e_{m I}=\frac{1}{c_{s}} \delta_{m n}-\frac{1-c_{s}^{2}}{c_{s}} \delta_{m 1} \delta_{n 1},
$$

where the adiabatic basis is defined as

$$
e_{1}^{I}=\sqrt{\frac{c_{s}}{2 X_{0}}} \dot{\phi}_{0}^{I} .
$$

Notice that the length of the basis vector $e_{1}^{I}$ is $c_{s}$ and that of the other basis vectors is $1 / c_{s}$. If we consider the two-field case $(I=1,2)$, the field perturbations are decomposed into the adiabatic field $Q_{\sigma}$ and the entropy field $Q_{s}$ as

$$
Q^{I}=Q_{\sigma} e_{1}^{I}+Q_{s} e_{2}^{I}
$$

Hereafter, for simplicity, we will concentrate on the two-field case although the extension to more fields is straightforward. 


\section{FOURTH-ORDER ACTION AND HAMILTONIAN AT LEADING ORDER}

We first calculate the fourth-order action for $Q_{n}$, where the subscript $n$ denotes either $\sigma$ or $s$. Since we are interested in the leading order shape of the trispectrum, we keep only the leading order in the slow-roll approximation, where the values of the slow-variation parameters defined by Eq. (10) are assumed to be small. We also assume $c_{s} \ll 1$ because otherwise the trispectrum is not observable in the future experiments. Using these approximations and following the ADM formalism [5, 17, 18, 91], the action up to fourth order can be calculated as

$$
\begin{aligned}
& S_{(2)}^{(\text {main })}=\frac{1}{2} \int d t d^{3} x \frac{a^{3}}{c_{s}^{2}}\left[\dot{Q}_{\sigma}^{2}+\dot{Q}_{s}^{2}-\frac{c_{s}^{2}}{a^{2}}\left(\partial_{i} Q_{\sigma} \partial^{i} Q_{\sigma}+\partial_{i} Q_{s} \partial^{i} Q_{s}\right)\right] \\
& S_{(3)}^{(\text {main })}=\frac{1}{2} \int d t d^{3} x \frac{a^{3}}{\sqrt{2 X_{0} c_{s}^{7}}}\left[\dot{Q}_{\sigma}^{3}+\dot{Q}_{\sigma} \dot{Q}_{s}^{2}+\frac{c_{s}^{2}}{a^{2}}\left(\left(\partial_{i} Q_{s} \partial^{i} Q_{s}-\partial_{i} Q_{\sigma} \partial^{i} Q_{\sigma}\right) \dot{Q}_{\sigma}-2\left(\partial_{i} Q_{\sigma} \partial^{i} Q_{s}\right) \dot{Q}_{s}\right)\right] \\
& S_{(4)}^{(\text {main })}= \frac{1}{16} \int d x^{3} d t \frac{a^{3}}{c_{s}^{5} X_{0}}\left[5 \dot{Q}_{\sigma}^{4}+6 \dot{Q}_{\sigma}^{2} \dot{Q}_{s}^{2}+\dot{Q}_{s}^{4}-\frac{2 c_{s}^{2}}{a^{2}}\left(3 \dot{Q}_{\sigma}^{2} \partial_{i} Q_{\sigma} \partial^{i} Q_{\sigma}-\dot{Q}_{\sigma}^{2} \partial_{i} Q_{s} \partial^{i} Q_{s}+4 \dot{Q}_{\sigma} \dot{Q}_{s} \partial_{i} Q_{\sigma} \partial^{i} Q_{s}\right.\right. \\
&\left.+\dot{Q}_{s}^{2} \partial_{i} Q_{\sigma} \partial^{i} Q_{\sigma}+\dot{Q}_{s}^{2} \partial_{i} Q_{s} \partial^{i} Q_{s}\right)(16) \\
&\left.+\frac{c_{s}^{4}}{a^{4}}\left(\left(\partial_{i} Q_{\sigma} \partial^{i} Q_{\sigma}\right)^{2}-2\left(\partial_{i} Q_{\sigma} \partial^{i} Q_{\sigma}\right)\left(\partial_{j} Q_{s} \partial^{j} Q_{s}\right)+4\left(\partial_{i} Q_{\sigma} \partial^{i} Q_{s}\right)^{2}+\left(\partial_{i} Q_{s} \partial^{i} Q_{s}\right)^{2}\right)\right]
\end{aligned}
$$

In general, the interaction Hamiltonian is not just the opposite sign of the interaction part of Lagrangian as is explained by the following calculation. Following 28], we define the Lagrangian density as

$$
\begin{aligned}
\mathcal{L}= & f_{a}^{(0)} \dot{\alpha}_{1}^{2}+f_{b}^{(0)} \dot{\alpha}_{2}^{2}+j^{(2)}+g_{a}^{(0)} \dot{\alpha}_{1}^{3}+g_{b}^{(0)} \dot{\alpha}_{1} \dot{\alpha}_{2}^{2}+g_{a}^{(2)} \dot{\alpha}_{1}+g_{b}^{(2)} \dot{\alpha}_{2}+j^{(3)} \\
& +h_{a}^{(0)} \dot{\alpha}_{1}^{4}+h_{b}^{(0)} \dot{\alpha}_{1}^{2} \dot{\alpha}_{2}^{2}+h_{c}^{(0)} \dot{\alpha}_{2}^{4}+h_{a}^{(2)} \dot{\alpha}_{1}^{2}+h_{b}^{(2)} \dot{\alpha}_{1} \dot{\alpha}_{2}+h_{c}^{(2)} \dot{\alpha}_{2}^{2}+j^{(4)}
\end{aligned}
$$

where $\alpha_{m}$ with $m=1,2$ denotes $Q_{\sigma}$ and $Q_{s}$ respectively. The $f$ 's, $j$ 's, $g$ 's and $h$ 's are all functions of $\alpha_{m}(t, \mathbf{x})$, its spatial derivative $\partial_{i} \alpha_{m}$ and time $t$. They come from 2 nd, 3rd and 4 th order action, respectively. For these functions, the superscripts (0), (1), (2), (3), (4), denote the order in the perturbations $\alpha_{m}$ of these functions. The subscripts $a$, $b, c$, just label the functions.

The momentum densities are given by

$$
\begin{aligned}
& \pi_{1} \equiv \frac{\partial \mathcal{L}}{\partial \dot{\alpha}_{1}}=2 f_{a}^{(0)} \dot{\alpha}_{1}+3 g_{a}^{(0)} \dot{\alpha}_{1}^{2}+g_{b}^{(0)} \dot{\alpha}_{2}^{2}+g_{a}^{(2)}+4 h_{a}^{(0)} \dot{\alpha}_{1}^{3}+2 h_{b}^{(0)} \dot{\alpha}_{1} \dot{\alpha}_{2}^{2}+2 h_{a}^{(2)} \dot{\alpha}_{1}+h_{b}^{(2)} \dot{\alpha}_{2} \\
& \pi_{2} \equiv \frac{\partial \mathcal{L}}{\partial \dot{\alpha}_{2}}=2 f_{b}^{(0)} \dot{\alpha}_{2}+2 g_{b}^{(0)} \dot{\alpha}_{1} \dot{\alpha}_{2}+g_{b}^{(2)}+2 h_{b}^{(0)} \dot{\alpha}_{1}^{2} \dot{\alpha}_{2}+4 h_{c}^{(0)} \dot{\alpha}_{2}^{3}+h_{b}^{(2)} \dot{\alpha}_{1}+2 h_{c}^{(2)} \dot{\alpha}_{2}
\end{aligned}
$$

Making use of these relations, $\dot{\alpha}_{m}$ are expressed in terms of $\pi_{m}$ up to third order as

$$
\dot{\alpha}_{1}=\frac{\pi_{1}}{2 f_{a}^{(0)}}+c_{a}^{(2)} \pi_{1}^{2}+c_{b}^{(2)} \pi_{2}^{2}+c_{a}^{(3)} \pi_{1}^{3}+c_{b}^{(3)} \pi_{1} \pi_{2}^{2}, \quad \dot{\alpha}_{2}=\frac{\pi_{2}}{2 f_{b}^{(0)}}+d_{a}^{(2)} \pi_{1} \pi_{2}+d_{a}^{(3)} \pi_{1}^{2} \pi_{2}+d_{b}^{(3)} \pi_{2}^{3},
$$

where the different $c$ 's and $d$ 's functions are given by

$$
\begin{aligned}
c_{a}^{(2)} & =-\frac{3 g_{a}^{(0)}}{8\left(f_{a}^{(0)}\right)^{3}}-\frac{g_{a}^{(2)}}{2 f_{a}^{(0)} \pi_{1}^{2}}, \quad c_{b}^{(2)}=-\frac{g_{b}^{(0)}}{8 f_{a}^{(0)}\left(f_{b}^{(0)}\right)^{2}}, \quad c_{a}^{(3)}=-\frac{1}{2 f_{a}^{(0)}}\left(\frac{3 g_{a}^{(0)} c_{a}^{(2)}}{f_{a}^{(0)}}+\frac{h_{a}^{(0)}}{2\left(f_{a}^{(0)}\right)^{3}}\right)-\frac{h_{a}^{(2)}}{2\left(f_{a}^{(0)}\right)^{2} \pi_{1}^{2}}, \\
c_{b}^{(3)} & \left.=-\frac{1}{2 f_{a}^{(0)}}\left(\frac{3 g_{a}^{(0)} c_{b}^{(2)}}{f_{a}^{(0)}}+\frac{g_{b}^{(0)} d_{a}^{(2)}}{f_{b}^{(0)}}+\frac{h_{b}^{(0)}}{4 f_{a}^{(0)}\left(f_{b}^{(0)}\right)^{2}}\right)-\frac{h_{b}^{(0)}}{4 f_{a}^{(0)} f_{b}^{(0)} \pi_{1} \pi_{2}}, \quad d_{a}^{(2)}=-\frac{g_{b}^{(2)}}{4 f_{a}^{(0)}\left(f_{b}^{(0)}\right)^{2}}-\frac{g_{b}^{(0)} \pi_{1} \pi_{2}}{2 f_{b}^{(2)}}\right)-\frac{h_{b}^{(2)}}{4 f_{a}^{(0)} f_{b}^{(0)} \pi_{1} \pi_{2}}, \\
d_{a}^{(3)} & =-\frac{1}{2 f_{b}^{(0)}}\left(\frac{g_{b}^{(0)} d_{a}^{(2)}}{f_{a}^{(0)}}+\frac{g_{b}^{(0)} c_{a}^{(2)}}{f_{b}^{(0)}}+\frac{h_{b}^{(0)}}{4\left(f_{a}^{(0)}\right)^{2} f_{b}^{(0)}}\right) \\
d_{b}^{(3)} & =-\frac{1}{2 f_{b}^{(0)}}\left(\frac{g_{b}^{(0)} c_{b}^{(2)}}{f_{b}^{(0)}}+\frac{h_{c}^{(0)}}{2\left(f_{b}^{(0)}\right)^{3}}\right)-\frac{h_{c}^{(2)}}{2\left(f_{b}^{(0)}\right)^{2} \pi_{2}^{2}}
\end{aligned}
$$


where again for these functions the superscripts (0), (1), (2), (3), denote the order in the perturbations $\alpha_{m}$ of these functions and the subscripts $a, b$, just label the functions.

The Hamiltonian density is obtained by plugging the previous expressions into

$$
\mathcal{H}=\pi_{1} \dot{\alpha}_{1}+\pi_{2} \dot{\alpha}_{2}-\mathcal{L} .
$$

We then separate $\mathcal{H}$ into a kinematic Hamiltonian density $\mathcal{H}_{0}$ which is given by

$$
\mathcal{H}_{0}=\frac{\pi_{1}^{2}}{4 f_{a}^{(0)}}+\frac{\pi_{2}^{2}}{4 f_{b}^{(0)}}-j^{(2)}
$$

and an interaction Hamiltonian density $\mathcal{H}^{\text {int }}$. To use the interaction picture formalism [94], $\alpha_{m}$ and $\pi_{m}$ in this interaction Hamiltonian should be replaced by their interaction picture counterparts $\alpha_{m}^{I}$ and $\pi_{m}^{I}$, which satisfy the free equation of motion determined by $\mathcal{H}_{0}$. They also satisfy the usual commutation relations

$$
\left[\alpha_{m}^{I}(t, \mathbf{x}), \pi_{n}^{I}(t, \mathbf{y})\right]=i \delta_{m n} \delta^{3}(\mathbf{x}-\mathbf{y}) .
$$

Expressing $\pi_{m}^{I}$ in $\mathcal{H}^{\text {int }}$ in terms of $\dot{\alpha}_{m}^{I}$ using

$$
\dot{\alpha}_{1}^{I}=\frac{\partial \mathcal{H}_{0}}{\partial \pi_{1}^{I}}=\frac{\pi_{1}^{I}}{2 f_{a}^{(0)}}, \quad \dot{\alpha}_{2}^{I}=\frac{\partial \mathcal{H}_{0}}{\partial \pi_{2}^{I}}=\frac{\pi_{2}^{I}}{2 f_{b}^{(0)}},
$$

we finally get the third-order and fourth-order interaction Hamiltonian densities as (omitting the label " $I$ " in the variables in $\mathcal{H}^{\text {int }}$ from now on)

$$
\begin{aligned}
\mathcal{H}_{(3)}^{i n t}= & -g_{a}^{(0)} \dot{\alpha}_{1}^{3}-g_{b}^{(0)} \dot{\alpha}_{1} \dot{\alpha}_{2}^{2}-g_{a}^{(2)} \dot{\alpha}_{1}-g_{b}^{(2)} \dot{\alpha}_{2}-j^{(3)} \\
\mathcal{H}_{(4)}^{i n t}= & \left(\frac{9\left(g_{a}^{(0)}\right)^{2}}{4 f_{a}^{(0)}}-h_{a}^{(0)}\right) \dot{\alpha}_{1}^{4}+\left(\frac{3 g_{a}^{(0)} g_{b}^{(0)}}{2 f_{a}^{(0)}}+\frac{\left(g_{b}^{(0)}\right)^{2}}{f_{b}^{(0)}}-h_{b}^{(0)}\right) \dot{\alpha}_{1}^{2} \dot{\alpha}_{2}^{2}+\left(\frac{\left(g_{b}^{(0)}\right)^{2}}{4 f_{a}^{(0)}}-h_{c}^{(0)}\right) \dot{\alpha}_{2}^{4} \\
& +\left(\frac{3 g_{a}^{(0)} g_{a}^{(2)}}{2 f_{a}^{(0)}}-h_{a}^{(2)}\right) \dot{\alpha}_{1}^{2}+\left(\frac{g_{b}^{(0)} g_{b}^{(2)}}{f_{b}^{(0)}}-h_{b}^{(2)}\right) \dot{\alpha}_{1} \dot{\alpha}_{2}+\left(\frac{g_{b}^{(0)} g_{a}^{(2)}}{2 f_{a}^{(0)}}-h_{c}^{(2)}\right) \dot{\alpha}_{2}^{2}+\left(\frac{\left(g_{a}^{(2)}\right)^{2}}{4 f_{a}^{(0)}}+\frac{\left(g_{b}^{(2)}\right)^{2}}{4 f_{b}^{(0)}}-j^{(4)}\right) .
\end{aligned}
$$

As in the single-field case [28], while the cubic part of $\mathcal{H}^{\text {int }}$ is the opposite sign of the cubic $\mathcal{L}^{\text {int }}$, this is generally not true at fourth order. As we will see, the extra terms contribute to the leading order results for $c_{s} \ll 1$. By applying Eq. (30) to the two-field DBI-inflation model given by Eqs. (15), (16) and (17), we can obtain the fourth-order interaction Hamiltonian density as

$$
\mathcal{H}_{(4)}^{i n t}=\frac{a^{3}}{4 X_{0} c_{s}^{5}} \dot{Q}_{\sigma}^{4}+\frac{a^{3}}{4 X_{0} c_{s}^{5}} \dot{Q}_{\sigma}^{2} \dot{Q}_{s}^{2}+\frac{a}{4 X_{0} c_{s}^{3}}\left(\partial_{i} Q_{s} \partial^{i} Q_{s}\right) \dot{Q}_{\sigma}^{2}+\frac{a}{4 X_{0} c_{s}^{3}}\left(\partial_{i} Q_{s} \partial^{i} Q_{s}\right) \dot{Q}_{s}^{2}
$$

\section{THE LEADING ORDER IN SLOW-ROLL FOUR-POINT FUNCTION}

In this section, we derive the connected four-point functions of the adiabatic and entropy fields coming from the intrinsic fourth-order contact interactions at leading order in the slow-roll expansion and in the small sound speed limit. The perturbations are promoted to quantum operators as

$$
Q_{n}(\tau, \mathbf{x})=\frac{1}{(2 \pi)^{3}} \int d^{3} \mathbf{k} Q_{n}(\tau, \mathbf{k}) e^{i \mathbf{k} \cdot \mathbf{x}}
$$

where $\tau$ denotes conformal time and

$$
Q_{n}(\tau, \mathbf{k})=u_{n}(\tau, \mathbf{k}) a_{n}(\mathbf{k})+u_{n}^{*}(\tau,-\mathbf{k}) a_{n}^{\dagger}(-\mathbf{k}),
$$

and $a_{n}(\mathbf{k})$ and $a_{n}^{\dagger}(-\mathbf{k})$ are the annihilation and creation operators, respectively, that satisfy the usual commutation relations:

$$
\left[a_{n}\left(\mathbf{k}_{\mathbf{1}}\right), a_{m}^{\dagger}\left(\mathbf{k}_{\mathbf{2}}\right)\right]=(2 \pi)^{3} \delta^{(3)}\left(\mathbf{k}_{\mathbf{1}}-\mathbf{k}_{\mathbf{2}}\right) \delta_{n m}, \quad\left[a_{n}\left(\mathbf{k}_{\mathbf{1}}\right), a_{m}\left(\mathbf{k}_{\mathbf{2}}\right)\right]=\left[a_{n}^{\dagger}\left(\mathbf{k}_{\mathbf{1}}\right), a_{m}^{\dagger}\left(\mathbf{k}_{\mathbf{2}}\right)\right]=0
$$


At leading order, the solutions for the mode functions are given by

$$
u_{n}(\tau, \mathbf{k})=A_{n} \frac{1}{k^{3 / 2}}\left(1+i k c_{s} \tau\right) e^{-i k c_{s} \tau} .
$$

The two-point correlation functions are then obtained as

$$
\left\langle 0\left|Q_{n}\left(\tau=0, \mathbf{k}_{\mathbf{1}}\right) Q_{m}\left(\tau=0, \mathbf{k}_{\mathbf{2}}\right)\right| 0\right\rangle=(2 \pi)^{3} \delta^{(3)}\left(\mathbf{k}_{\mathbf{1}}+\mathbf{k}_{\mathbf{2}}\right) \mathcal{P}_{Q_{n}} \frac{2 \pi^{2}}{k_{1}^{3}} \delta_{n m},
$$

where the power spectra $\mathcal{P}_{Q_{n}}$ are defined as

$$
\mathcal{P}_{Q_{n}}=\frac{\left|A_{n}\right|^{2}}{2 \pi^{2}}, \quad\left|A_{\sigma}\right|^{2}=\left|A_{s}\right|^{2}=\frac{H^{2}}{2 c_{s}},
$$

and they should be evaluated at the time of the sound horizon crossing $c_{s_{*}} k_{1}=a_{*} H_{*}$.

In terms of these quantum operators, the connected four-point correlation function coming from the contact interaction in the interaction picture formalism is given by [5, 94]

$$
\left\langle\Omega\left|Q_{m}\left(t, \mathbf{k}_{\mathbf{1}}\right) Q_{n}\left(t, \mathbf{k}_{\mathbf{2}}\right) Q_{p}\left(t, \mathbf{k}_{\mathbf{3}}\right) Q_{q}\left(t, \mathbf{k}_{\mathbf{4}}\right)\right| \Omega\right\rangle=-i \int_{t_{0}}^{t} d \tilde{t}\left\langle 0\left|\left[Q_{m}\left(t, \mathbf{k}_{\mathbf{1}}\right) Q_{n}\left(t, \mathbf{k}_{\mathbf{2}}\right) Q_{p}\left(t, \mathbf{k}_{\mathbf{3}}\right) Q_{q}\left(t, \mathbf{k}_{\mathbf{4}}\right), H^{i n t}(\tilde{t})\right]\right| 0\right\rangle,
$$

where $t_{0}$ is some early time during inflation when the fields' vacuum fluctuations are deep inside the horizon and $t$ is some time after the horizon exit. $|\Omega\rangle$ is the interacting vacuum which is different from the free theory vacuum $|0\rangle$. If one uses conformal time, it is a good approximation to perform the integration from $-\infty$ to 0 because $\tau \approx-(a H)^{-1}$. The interaction Hamiltonian $H^{\text {int }}$ is given by $H^{i n t}=\int d^{3} x \mathcal{H}^{\text {int }}$.

The purely adiabatic, purely entropic and mixed components are given by

$$
\begin{gathered}
\left\langle\Omega\left|Q_{\sigma}\left(0, \mathbf{k}_{\mathbf{1}}\right) Q_{\sigma}\left(0, \mathbf{k}_{\mathbf{2}}\right) Q_{\sigma}\left(0, \mathbf{k}_{\mathbf{3}}\right) Q_{\sigma}\left(0, \mathbf{k}_{\mathbf{4}}\right)\right| \Omega\right\rangle=(2 \pi)^{3} \delta^{(3)}\left(\sum_{i=1}^{4} \mathbf{k}_{\mathbf{i}}\right) \frac{H^{8}}{2 X_{0} c_{s}^{6}} \frac{1}{\Pi_{i=1}^{4} k_{i}^{3}}\left(-36 A_{1}\right), \\
A_{1}=\frac{\Pi_{i=1}^{4} k_{i}^{2}}{K^{5}}, \quad K=\sum_{i=1}^{4} k_{i} \\
\left\langle\Omega\left|Q_{s}\left(0, \mathbf{k}_{\mathbf{1}}\right) Q_{s}\left(0, \mathbf{k}_{\mathbf{2}}\right) Q_{s}\left(0, \mathbf{k}_{\mathbf{3}}\right) Q_{s}\left(0, \mathbf{k}_{\mathbf{4}}\right)\right| \Omega\right\rangle=(2 \pi)^{3} \delta^{(3)}\left(\sum_{i=1}^{4} \mathbf{k}_{\mathbf{i}}\right) \frac{H^{8}}{2 X_{0} c_{s}^{6}} \frac{1}{\Pi_{i=1}^{4} k_{i}^{3}}\left(-\frac{1}{8} A_{2}\right), \\
A_{2}=\frac{k_{1}^{2} k_{2}^{2}\left(\mathbf{k}_{\mathbf{3}} \cdot \mathbf{k}_{\mathbf{4}}\right)}{K^{3}}\left(1+\frac{3\left(k_{3}+k_{4}\right)}{K}+\frac{12 k_{3} k_{4}}{K^{2}}\right)+\text { perm. } \\
\left\langle\Omega\left|Q_{\sigma}\left(0, \mathbf{k}_{\mathbf{1}}\right) Q_{\sigma}\left(0, \mathbf{k}_{\mathbf{2}}\right) Q_{s}\left(0, \mathbf{k}_{\mathbf{3}}\right) Q_{s}\left(0, \mathbf{k}_{\mathbf{4}}\right)\right| \Omega\right\rangle=(2 \pi)^{3} \delta^{(3)}\left(\sum_{i=1}^{4} \mathbf{k}_{\mathbf{i}}\right) \frac{H^{8}}{2 X_{0} c_{s}^{6}} \frac{-1}{\Pi_{i=1}^{4} k_{i}^{3}}\left(6 A_{1}+\frac{1}{2} A_{3}\right), \\
A_{3}=\frac{k_{1}^{2} k_{2}^{2}\left(\mathbf{k}_{\mathbf{3}} \cdot \mathbf{k}_{\mathbf{4}}\right)}{K^{3}}\left(1+\frac{3\left(k_{3}+k_{4}\right)}{K}+\frac{12 k_{3} k_{4}}{K^{2}}\right),
\end{gathered}
$$

where "perm." in Eq. (42) denotes the 23 permutations of the four-momenta. The purely adiabatic component agrees with the result of the single-field DBI-inflation model [28].

We need to relate the four-point functions of the scalar fields to the four-point function of the comoving curvature perturbation $\mathcal{R}$ which is closely related to the observable quantity. As in [35], $\mathcal{R}$ and $Q_{\sigma}$ are related as

$$
\mathcal{R}=\frac{\sqrt{c_{s}} H}{\sqrt{2 X_{0}}} Q_{\sigma},
$$

and it is convenient to define the entropy perturbation $\mathcal{S}$ as

$$
\mathcal{S}=\frac{\sqrt{c_{s}} H}{\sqrt{2 X_{0}}} Q_{s}
$$

so that the power spectra are $\mathcal{P}_{\mathcal{S}_{*}} \simeq \mathcal{P}_{\mathcal{R}_{*}}$, where the subscript $*$ means that the quantity should be evaluated at the sound horizon crossing. 
In this work, we ignore the possibility that the entropy perturbation during inflation can lead to a primordial entropy perturbation that could be observable in the CMB. But we shall consider the effect of the entropy perturbation on the final curvature perturbation. Following the analysis of [95], we describe the conversion of the entropy perturbation into the curvature perturbation by a transfer coefficient $T_{\mathcal{R} \mathcal{S}}$. Then the final curvature perturbation is expressed in terms of the adiabatic and entropy field perturbations as

$$
\mathcal{R}=\mathcal{A}_{\sigma} Q_{\sigma *}+\mathcal{A}_{s} Q_{s *}, \quad \mathcal{A}_{\sigma}=\left(\frac{\sqrt{c_{s}} H}{\sqrt{2 X_{0}}}\right)_{*}, \quad \mathcal{A}_{s}=T_{\mathcal{R S}}\left(\frac{\sqrt{c_{s}} H}{\sqrt{2 X_{0}}}\right)_{*} .
$$

Hence the connected four-point function of $\mathcal{R}$ at leading order is given by

$$
\begin{aligned}
\left\langle\mathcal{R}\left(\mathbf{k}_{\mathbf{1}}\right) \mathcal{R}\left(\mathbf{k}_{\mathbf{2}}\right) \mathcal{R}\left(\mathbf{k}_{\mathbf{3}}\right) \mathcal{R}\left(\mathbf{k}_{\mathbf{4}}\right)\right\rangle= & \mathcal{A}_{\sigma}^{4}\left\langle Q_{\sigma}\left(\mathbf{k}_{\mathbf{1}}\right) Q_{\sigma}\left(\mathbf{k}_{\mathbf{2}}\right) Q_{\sigma}\left(\mathbf{k}_{\mathbf{3}}\right) Q_{\sigma}\left(\mathbf{k}_{\mathbf{4}}\right)\right\rangle+\mathcal{A}_{\sigma}^{2} \mathcal{A}_{s}^{2}\left(\left\langle Q_{\sigma}\left(\mathbf{k}_{\mathbf{1}}\right) Q_{\sigma}\left(\mathbf{k}_{\mathbf{2}}\right) Q_{s}\left(\mathbf{k}_{\mathbf{3}}\right) Q_{s}\left(\mathbf{k}_{\mathbf{4}}\right)\right\rangle+\operatorname{perm} .\right) \\
& +\mathcal{A}_{s}^{4}\left\langle Q_{s}\left(\mathbf{k}_{\mathbf{1}}\right) Q_{s}\left(\mathbf{k}_{\mathbf{2}}\right) Q_{s}\left(\mathbf{k}_{\mathbf{3}}\right) Q_{s}\left(\mathbf{k}_{\mathbf{4}}\right)\right\rangle,
\end{aligned}
$$

where "perm" denotes five permutations of the four-momenta. This constitutes one of the main results of this work.

Since the mixed component and the purely entropic component have different momentum dependence from the purely adiabatic component, the momentum dependence of the resultant four-point function of the curvature perturbation is different from that in the single-field DBI-inflation model [28]. The only effect for the bispectrum due to the presence of the multiple fields is a change in its amplitude with respect to the single field case, however for the trispectrum the presence of the multiple fields affects also the shape dependence. So in principle, the trispectrum can be used to distinguish between the multi-field and the single-field DBI-inflation models.

\section{LORENTZ BOOST}

In this section, we will present an alternative and simpler method to obtain the leading order ${ }^{1}$ action for the perturbations.

It is well known that the DBI action (11) describes the motion of a brane in a higher dimensional spacetime. For simplicity, let us take $g_{\mu \nu}$ as the Minkowski metric ${ }^{2}$ and $f$ is constant. Then in the frame where the brane is at rest in the background (brane-rest frame), $\tilde{X}$ will be a small quantity because it is written in terms of perturbations of the brane positions. Then we can expand the Lagrangian (11), by ignoring the potential terms which do not contribute at the leading order as

$$
\tilde{P}=X-\frac{f}{2} X^{2}+f X_{I}^{J} X_{J}^{I}+\mathcal{O}\left(X_{I J}^{3}\right)
$$

where $X$ and $X_{I J}$ are written in the coordinates of this new frame that we will denote by $(\tilde{t}, \mathbf{x}, \tilde{\sigma}, s)$. $\tilde{\sigma}$ is the coordinate along the direction of the motion of the brane in the background (adiabatic direction) and $s$ parameterizes the orthogonal direction (entropy direction). In the brane-rest frame, the DBI action for the two-field model can be written in a fairly simple form as

$$
S_{\text {rest }}=\int\left[-\frac{1}{2} \partial_{\mu} \tilde{\sigma} \partial^{\mu} \tilde{\sigma}-\frac{1}{2} \partial_{\mu} s \partial^{\mu} s+\frac{f}{8}\left(\left(\partial_{\mu} \tilde{\sigma} \partial^{\mu} \tilde{\sigma}\right)^{2}+\left(\partial_{\mu} s \partial^{\mu} s\right)^{2}+4\left(\partial_{\mu} s \partial^{\mu} \tilde{\sigma}\right)^{2}-2 \partial_{\mu} \tilde{\sigma} \partial^{\mu} \tilde{\sigma} \partial_{\nu} s \partial^{\nu} s\right)\right] d \tilde{t} d \mathbf{x},(50)
$$

where we have ignored higher-order terms.

We are interested in the behaviour of the perturbations in the frame corresponding to the set-up shown in the previous sections and for this purpose, it is necessary to know the Lagrangian of the perturbations in such a frame. From Eq. (8), this frame is the one where the brane is moving with the velocity $v=\sqrt{2 f X_{0}}$ in the background (brane-moving frame). If we label the coordinates of the brane-moving frame as $(t, \mathbf{x}, \sigma, s)$, the coordinate variables in these two frames are related by a Lorentz transformation

$$
t=\gamma(\tilde{t}+v \sqrt{f} \tilde{\sigma}), \quad \sqrt{f} \sigma=\gamma(\sqrt{f} \tilde{\sigma}+v \tilde{t})
$$

\footnotetext{
${ }^{1}$ In fact, with this method one can also obtain the sub-leading terms in $c_{s}^{2}$.

${ }^{2}$ In our case $g_{\mu \nu}$ will be an inflating FRW metric but this is conformally Minkowski and the final result will be the same as for the Minkowski case, up to powers of the scale factor.
} 
and its inverse transformation

$$
\tilde{t}=\gamma(t-v \sqrt{f} \sigma), \quad \sqrt{f} \tilde{\sigma}=\gamma(\sqrt{f} \sigma-v t)
$$

with $\mathbf{x}$ and $s$ unchanged. From Eq. (51), in terms of the background value $\sigma_{0}, v$ can be expressed as $v^{2}=f \dot{\sigma}_{0}^{2}$, which means $\sigma_{0}=\sqrt{2 X_{0}}$. We are interested in the small sound speed limit or equivalently when the brane is relativistic, i.e. $v \sim 1$.

In order to see the behaviour of the perturbations in the brane-moving frame, it is convenient to introduce a new variable as

$$
\delta \sigma(t, \mathbf{x}) \equiv \sigma(t, \mathbf{x})-\frac{v t}{\sqrt{f}}=\frac{\tilde{\sigma}(\tilde{t}, \mathbf{x})}{\gamma}
$$

which is nothing but the adiabatic perturbation in the brane-moving frame, since $v t / \sqrt{f}$ can be interpreted as $\sigma_{0}$ in the case $\dot{\sigma}_{0}$ is constant. For the entropy perturbation in the brane-moving frame, we can continue to use $s$, since $s$ is invariant under the Lorentz transformation. It is worth noting that these $\delta \sigma$ and $s$ are related with $Q_{\sigma}$ and $Q_{s}$ introduced in Eq. (14) as

$$
\delta \sigma=\sqrt{c_{s}} Q_{\sigma}, \quad s=\frac{1}{\sqrt{c_{s}}} Q_{s},
$$

since $Q_{\sigma}$ and $Q_{s}$ are defined in terms of the basis satisfying (12) and (13).

For the Lagrangian in the brane-moving frame it is necessary to know the transformation law of not only the coordinate variables, but also its derivatives. Since the time derivative of a quantity $q$ in the brane-moving frame is

$$
\frac{\partial q}{\partial t}(t, \mathbf{x})=\frac{\partial q}{\partial \tilde{t}}(\tilde{t}, \mathbf{x}) \frac{\partial \tilde{t}}{\partial t}=\frac{\partial q}{\partial \tilde{t}}(\tilde{t}, \mathbf{x})\left(\frac{1}{\gamma}-v \sqrt{f} \gamma \delta \dot{\sigma}\right)=\tilde{\gamma}^{-1} \frac{\partial q}{\partial \tilde{t}}(\tilde{t}, \mathbf{x})
$$

where we define $\tilde{\gamma}$ as

$$
\tilde{\gamma}=\left(\frac{1}{\gamma}-v \sqrt{f} \gamma \delta \dot{\sigma}\right)^{-1} \approx \gamma\left(1+v \sqrt{f} \gamma^{2} \delta \dot{\sigma}+v^{2} f \gamma^{4}(\delta \dot{\sigma})^{2}\right)+\mathcal{O}\left((\delta \dot{\sigma})^{3}\right),
$$

we can express the time derivative of a quantity $q$ in the brane-rest frame by the one in the brane-moving frame as

$$
\frac{\partial q}{\partial \tilde{t}}(\tilde{t}, \mathbf{x})=\tilde{\gamma} \frac{\partial q}{\partial t}(t, \mathbf{x})
$$

Similarly, the spatial gradient of a quantity $q$ in the brane-rest frame can be related with the one in the brane-moving frame as

$$
\nabla q(\tilde{t}, \mathbf{x})=\nabla q(t, \mathbf{x})+v \sqrt{f} \gamma \tilde{\gamma} \nabla(\delta \sigma) \dot{q}
$$

One can now use Eqs. (50), (52), (54), (57) and (58) to obtain the actions up to fourth-order in the brane-moving frame as

$$
\begin{gathered}
S_{m o v}^{(2)} \sim \int \frac{a^{3}}{2 c_{s}^{2}}\left[\dot{Q}_{\sigma}^{2}+\dot{Q}_{s}^{2}-\frac{c_{s}^{2}}{a^{2}}\left(\partial_{i} Q_{\sigma} \partial^{i} Q_{\sigma}+\partial_{i} Q_{s} \partial^{i} Q_{s}\right)\right] d t d \mathbf{x} \\
S_{m o v}^{(3)} \sim \int \frac{a^{3}}{2 \sqrt{2 X_{0} c_{s}^{7}}}\left[\dot{Q}_{\sigma}^{3}+\dot{Q}_{\sigma} \dot{Q}_{s}^{2}-\frac{c_{s}^{2}}{a^{2}}\left\{\dot{Q}_{\sigma}\left(\partial_{i} Q_{\sigma} \partial^{i} Q_{\sigma}-\partial_{i} Q_{s} \partial^{i} Q_{s}\right)+2 \dot{Q}_{s} \partial_{i} Q_{\sigma} \partial^{i} Q_{s}\right\}\right] d t d \mathbf{x}, \\
S_{m o v}^{(4)} \sim \int \frac{a^{3}}{16 c_{s}^{5} X_{0}}\left[5 \dot{Q}_{\sigma}^{4}+6 \dot{Q}_{\sigma}^{2} \dot{Q}_{s}^{2}+\dot{Q}_{s}^{4}\right. \\
-\frac{2 c_{s}^{2}}{a^{2}}\left\{3 \dot{Q}_{\sigma}^{2} \partial_{i} Q_{\sigma} \partial^{i} Q_{\sigma}-\dot{Q}_{\sigma}^{2} \partial_{i} Q_{s} \partial^{i} Q_{s}+4 \dot{Q}_{\sigma} \dot{Q}_{s} \partial_{i} Q_{\sigma} \partial^{i} Q_{s}+\dot{Q}_{s}^{2} \partial_{i} Q_{\sigma} \partial^{i} Q_{\sigma}+\dot{Q}_{s}^{2} \partial_{i} Q_{s} \partial^{i} Q_{s}\right\} \\
\left.+\frac{c_{s}^{4}}{a^{4}}\left\{\left(\partial_{i} Q_{\sigma} \partial^{i} Q_{\sigma}\right)^{2}-2\left(\partial_{i} Q_{\sigma} \partial^{i} Q_{\sigma}\right)\left(\partial_{i} Q_{s} \partial^{i} Q_{s}\right)+4\left(\partial_{i} Q_{\sigma} \partial^{i} Q_{s}\right)^{2}+\left(\partial_{i} Q_{s} \partial^{i} Q_{s}\right)^{2}\right\}\right] d t d \mathbf{x}
\end{gathered}
$$


where we introduced the scale factor dependence, we used $d \tilde{t}=d t / \tilde{\gamma}, v \sim 1$ and $f \sim 1 / \dot{\sigma}_{0}^{2} \sim 1 /\left(2 X_{0}\right)$. Although cubic interactions are absent in the brane-rest frame (see Eq. (50)), they are induced by the boost in the brane-moving frame (see Eq. (60) ). At fourth order, quartic interactions are already present in the brane-rest frame but additional terms are induced in the brane-moving frame. It is worth noting that Eqs. (59), (60) and (61) agree with Eqs. (15), (16) and (17) derived by the usual ADM formalism.

Now we are in the position to discuss the momentum dependence of the bispectrum and trispectrum. The secondorder action in the rest frame of the brane is symmetric under the exchange between the adiabatic and entropy modes. At quadratic order, because of the change of the time coordinate, $d t \approx \gamma d \tilde{t}$, the sound speed in the brane-moving frame deviates from the speed of light. Since the shift of the sound speed is solely due to this coordinate change, both the adiabatic and entropy modes should have a common sound speed [37. The third-order action in the branemoving frame, which is generated by the boost transformation, also originates from the second-order action in the brane rest frame. As is seen from Eqs. (57) and (58), the boost acts on both modes in the same way. Therefore the boost transformation preserves the symmetry between the adiabatic and entropy modes in the sense that the mixed component which contains $Q_{s}$ reduces to the purely adiabatic component if all $Q_{s}$ are replaced with $Q_{\sigma}$. At third order, the interaction Hamiltonian is just the minus of the third-order interaction Lagrangian. Thus, at this order, the three-point function from the mixed component has the same momentum dependence as in the purely adiabatic case. Thus, adding the contributions from the entropy modes does not change the momentum dependence.

At fourth order, there are two contributions to the action in the brane-moving frame. One is the fourth-order action that arises by the boost from the second-order action in the brane-rest frame:

$$
S_{\text {boost }}^{(4)} \sim \int \frac{a^{3}}{4 c_{s}^{5} X_{0}}\left[\dot{Q}_{\sigma}^{4}+\dot{Q}_{\sigma}^{2} \dot{Q}_{s}^{2}-\frac{c_{s}^{2}}{a^{2}}\left(\dot{Q}_{\sigma}^{2} \partial_{i} Q_{\sigma} \partial^{i} Q_{\sigma}+\dot{Q}_{s}^{2} \partial_{i} Q_{\sigma} \partial^{i} Q_{\sigma}\right)\right] d t d \mathbf{x} .
$$

As in the case of the third-order action, the boost preserves the symmetry between the adiabatic and entropy modes. Thus, the mixed component becomes the same as the pure adiabatic component by replacing $Q_{s}$ with $Q_{\sigma}$ and the mixed component would give the same shape dependence of the trispectrum as the pure adiabatic component. However, at fourth order, there exists the intrinsic fourth-order action in the brane-rest frame which contains pure adiabatic, pure entropy and mixed components. If one adds these contributions to Eq. (62), the symmetry between the adiabatic mode and entropy mode is broken. Moreover, at fourth order, the interaction Hamiltonian is not simply the opposite sign of the fourth-order Lagrangian and the additional contributions in the interaction Hamiltonian also do not have the symmetry between the adiabatic mode and entropy mode. Thus the momentum dependence of the trispectrum becomes different for adiabatic, entropy and mixed components.

Therefore the third order is a special case where the shape of the bispectrum is not changed by the entropy modes. This is because, at third order, the interaction Hamiltonian only arises by the boost which preserves the symmetry between the adiabatic and the entropy modes. At higher orders, we expect that the momentum dependence of the $n$-point function induced by the entropy modes is different from the pure adiabatic contribution. This would be crucial to distinguish between single field and multi-field models by the shape dependence of the $n$-point functions.

\section{CONCLUSIONS}

In the multi-field DBI-inflation model, it had been shown that the sound speeds for the adiabatic and entropy perturbations are the same [36]. It was also shown that the momentum dependence of the three-point function of the final curvature perturbation remains the same as in the single-field case, that is, the components of the three-point function including the entropy perturbations only change the amplitude of the three-point function from the purely adiabatic component. This is because there exists a symmetry under the exchange between the adiabatic and entropy modes in the second- and third-order actions for $Q_{n}$ at leading order in slow-roll and in the small sound speed limit.

In this paper, as a natural extension of these works, we studied the non-Gaussianity from the contact interaction trispectrum in the multi-field DBI-inflation model. We first derived the fourth-order action for the perturbations based on the usual ADM formalism (Eq.(17)). It is written in terms of the adiabatic and entropy perturbations in the small sound speed limit and at leading order in the slow-roll expansion.

After deriving the relation between the interaction Lagrangian and the interaction Hamiltonian, which can be applied to a fairly general two-field model, we obtained the fourth-order interaction Hamiltonian (Eq. (31)). It is worth noting that while the cubic part of the interaction Hamiltonian is the opposite sign of the Lagrangian density, this is generally not true at higher orders [28].

Using this interaction Hamiltonian, we derived the connected four-point function coming from the intrinsic fourthorder contact interaction, in the small sound speed limit and at leading order in the slow-roll expansion. In these approximations, in addition to the purely adiabatic four-point function $\left\langle Q_{\sigma}\left(\mathbf{k}_{\mathbf{1}}\right) Q_{\sigma}\left(\mathbf{k}_{\mathbf{2}}\right) Q_{\sigma}\left(\mathbf{k}_{\mathbf{3}}\right) Q_{\sigma}\left(\mathbf{k}_{\mathbf{4}}\right)\right\rangle$, there exists a purely entropic component $\left\langle Q_{s}\left(\mathbf{k}_{\mathbf{1}}\right) Q_{s}\left(\mathbf{k}_{\mathbf{2}}\right) Q_{s}\left(\mathbf{k}_{\mathbf{3}}\right) Q_{s}\left(\mathbf{k}_{\mathbf{4}}\right)\right\rangle$ and a mixed component $\left\langle Q_{\sigma}\left(\mathbf{k}_{\mathbf{1}}\right) Q_{\sigma}\left(\mathbf{k}_{\mathbf{2}}\right) Q_{s}\left(\mathbf{k}_{\mathbf{3}}\right) Q_{s}\left(\mathbf{k}_{\mathbf{4}}\right)\right\rangle$. It 
was shown that the purely entropic and the mixed components have different momentum dependence from the purely adiabatic component (Eqs. (39), (41) and (43)). Because of this it was shown that the momentum dependence of the four-point function of the comoving curvature perturbations is affected by the entropy modes (Eq. (48)), and the shape is different from the single-field case. In contrast to the shape of the bispectrum, which does not distinguish the multi-field DBI-inflation model from the single-field DBI-inflation model, the CMB trispectrum can provide a useful discriminator for the multi-field DBI-inflation model.

We also derived the fourth-order action for the perturbations by an alternative and simpler method. Since the DBI action describes the motion of a brane in a higher dimensional spacetime, the action for the perturbations can be obtained by a Lorentz boost from the frame where the brane is at rest in the background to the frame where the brane moves at the velocity $v$ with $v^{2}=2 f_{0} X_{0}=f_{0} \dot{\sigma}_{0}^{2}$. In the small sound speed limit $(v \rightarrow 1)$, the actions up to fourth order in the frame where the brane is moving were calculated (Eqs. (59), (60) and (61)) and it was shown that they coincide with the ones obtained by the usual method (Eqs. (15), (16) and (17)). From this derivation, we found that, at third order, the interaction Hamiltonian arises purely by the boost and it has the symmetry under the exchange between the adiabatic mode and entropy mode. This is because the boost does not distinguish between them. At fourth order, there exists the intrinsic fourth-order action in the rest frame of the brane. This breaks the symmetry. In addition, the interaction Hamiltonian is not just the opposite sign of the Lagrangian. The additional terms in the interaction Hamiltonian also break the symmetry. Hence the trispectrum coming from the entropy modes has a different shape dependence from the one coming from the adiabatic modes.

In order to calculate the effect of the entropy perturbation on the curvature perturbation, we need to specify a model that describes how the entropy perturbation is converted to the curvature perturbation. In this paper, we modeled this transfer by a transfer function $T_{\mathcal{R} \mathcal{S}}$. It would be interesting to study this mixing in specific string theory motivated models.

In this paper, we considered the trispectrum coming from the intrinsic fourth-order contact interaction. However, as it was shown recently in [55, 85, 86], there are other important contributions for the trispectrum coming from the interactions at a distance such as the exchange of scalar particles. In particular, Ref. [55] calculated a part of the trispectrum coming from the entropy modes, i.e. $\left\langle\mathcal{R}\left(\mathbf{k}_{\mathbf{1}}\right) \mathcal{R}\left(\mathbf{k}_{\mathbf{2}}\right) \mathcal{R}\left(\mathbf{k}_{\mathbf{3}}\right) \mathcal{R}\left(\mathbf{k}_{\mathbf{4}}\right)\right\rangle \propto T_{\mathcal{R} \mathcal{S}}^{4}\left\langle Q_{s}\left(\mathbf{k}_{\mathbf{1}}\right) Q_{s}\left(\mathbf{k}_{\mathbf{2}}\right) Q_{s}\left(\mathbf{k}_{\mathbf{3}}\right) Q_{s}\left(\mathbf{k}_{\mathbf{4}}\right)\right\rangle$, when an adiabatic scalar particle is exchanged. We will present the full leading order trispectrum in the multi-field DBI-inflation model in a separate publication [96].

\section{Acknowledgments}

SM and FA are supported by the Japanese Society for the Promotion of Science (JSPS). KK is supported by ERC, RCUK and STFC. TT is supported by Grants-in-Aid for Scientific Research, Nos. 19540285, 17340075 and 21244033. SM is grateful to the ICG, Portsmouth for their hospitality when part of this work was done. The authors also would like to mention that discussions during the GCOE/YITP workshop YITP-W-09-01 on "Non-linear cosmological perturbations" were useful to complete this work.

[1] http://map.gsfc.nasa.gov/

[2] A. P. S. Yadav and B. D. Wandelt, Phys. Rev. Lett. 100, 181301 (2008), 0712.1148.

[3] WMAP, E. Komatsu et al., Astrophys. J. Suppl. 180, 330 (2009), 0803.0547.

[4] K. M. Smith, L. Senatore, and M. Zaldarriaga, (2009), 0901.2572.

[5] J. M. Maldacena, JHEP 0305 (2003) 013 arXiv:astro-ph/0210603.

[6] http://www.rssd.esa.int/index.php?project=Planck

[7] A. D. Linde and V. F. Mukhanov, Phys. Rev. D56, 535 (1997), astro-ph/9610219.

[8] N. Bartolo, S. Matarrese, and A. Riotto, Phys. Rev. D65, 103505 (2002), hep-ph/0112261

[9] F. Bernardeau and J.-P. Uzan, Phys. Rev. D66, 103506 (2002), hep-ph/0207295.

[10] F. Bernardeau and J.-P. Uzan, Phys. Rev. D67, 121301 (2003), astro-ph/0209330

[11] G. Dvali, A. Gruzinov, and M. Zaldarriaga, Phys. Rev. D69, 023505 (2004), astro-ph/0303591.

[12] P. Creminelli, JCAP 0310, 003 (2003), astro-ph/0306122.

[13] M. Alishahiha, E. Silverstein and D. Tong, Phys. Rev. D 70 (2004) 123505 arXiv:hep-th/0404084.

[14] A. Gruzinov, Phys. Rev. D71, 027301 (2005), astro-ph/0406129

[15] N. Bartolo, E. Komatsu, S. Matarrese and A. Riotto, Phys. Rept. 402, 103 (2004) arXiv:astro-ph/0406398.

[16] K. Enqvist, A. Jokinen, A. Mazumdar, T. Multamaki, and A. Vaihkonen, Phys. Rev. Lett. 94, 161301 (2005), astro-ph/0411394.

[17] D. Seery and J. E. Lidsey, JCAP 0506 (2005) 003 arXiv:astro-ph/0503692.

[18] D. Seery and J. E. Lidsey, JCAP 0509 (2005) 011 arXiv:astro-ph/0506056. 
[19] A. Jokinen and A. Mazumdar, JCAP 0604, 003 (2006), astro-ph/0512368

[20] D. H. Lyth, JCAP 0511, 006 (2005), astro-ph/0510443.

[21] M. P. Salem, Phys. Rev. D72, 123516 (2005), astro-ph/0511146

[22] D. Seery and J. E. Lidsey, JCAP 0701, 008 (2007), astro-ph/0611034.

[23] M. Sasaki, J. Valiviita, and D. Wands, Phys. Rev. D74, 103003 (2006), astro-ph/0607627

[24] K. A. Malik and D. H. Lyth, JCAP 0609, 008 (2006), astro-ph/0604387.

[25] N. Barnaby and J. M. Cline, Phys. Rev. D73, 106012 (2006), astro-ph/0601481.

[26] L. Alabidi and D. Lyth, JCAP 0608, 006 (2006), astro-ph/0604569.

[27] X. Chen, M. x. Huang, S. Kachru and G. Shiu, JCAP 0701 (2007) 002 arXiv:hep-th/0605045.

[28] X. Chen, M. x. Huang and G. Shiu, Phys. Rev. D 74 (2006) 121301 arXiv:hep-th/0610235.

[29] X. Chen, R. Easther, and E. A. Lim, JCAP 0706, 023 (2007), astro-ph/0611645

[30] L. Alabidi, JCAP 0610, 015 (2006), astro-ph/0604611.

[31] D. Seery, J. E. Lidsey and M. S. Sloth, JCAP 0701, 027 (2007) arXiv:astro-ph/0610210].

[32] C. T. Byrnes, M. Sasaki, and D. Wands, Phys. Rev. D74, 123519 (2006), astro-ph/0611075

[33] T. Suyama and M. Yamaguchi, Phys. Rev. D77, 023505 (2008), 0709.2545.

[34] F. Arroja and K. Koyama, Phys. Rev. D 77, 083517 (2008) arXiv:0802.1167 [hep-th]].

[35] F. Arroja, S. Mizuno and K. Koyama, JCAP 0808, 015 (2008) arXiv:0806.0619 [astro-ph]].

[36] D. Langlois, S. Renaux-Petel, D. A. Steer and T. Tanaka, Phys. Rev. Lett. 101, 061301 (2008) arXiv:0804.3139 [hep-th]].

[37] D. Langlois, S. Renaux-Petel, D. A. Steer and T. Tanaka, Phys. Rev. D 78, 063523 (2008) arXiv:0806.0336 [hep-th]].

[38] D. Seery, M. S. Sloth and F. Vernizzi, JCAP 0903, 018 (2009) arXiv:0811.3934 [astro-ph]].

[39] M. Sasaki, Prog. Theor. Phys. 120, 159 (2008), 0805.0974.

[40] C. T. Byrnes, K.-Y. Choi, and L. M. H. Hall, JCAP 0810, 008 (2008), 0807.1101.

[41] C. T. Byrnes, K.-Y. Choi, and L. M. H. Hall, JCAP 0902, 017 (2009), 0812.0807.

[42] B. Dutta, L. Leblond, and J. Kumar, Phys. Rev. D78, 083522 (2008), 0805.1229.

[43] A. Naruko and M. Sasaki, Prog. Theor. Phys. 121, 193 (2009), 0807.0180.

[44] T. Suyama and F. Takahashi, JCAP 0809, 007 (2008), 0804.0425.

[45] X. Gao, JCAP 0806, 029 (2008) arXiv:0804.1055 [astro-ph]].

[46] H. R. S. Cogollo, Y. Rodriguez, and C. A. Valenzuela-Toledo, JCAP 0808, 029 (2008), 0806.1546.

[47] Y. Rodriguez and C. A. Valenzuela-Toledo, (2008), 0811.4092.

[48] K. Ichikawa, T. Suyama, T. Takahashi, and M. Yamaguchi, Phys. Rev. D78, 023513 (2008), 0802.4138.

[49] C. T. Byrnes, JCAP 0901, 011 (2009), 0810.3913.

[50] S. Li, Y.-F. Cai, and Y.-S. Piao, Phys. Lett. B671, 423 (2009), 0806.2363.

[51] D. Langlois, F. Vernizzi, and D. Wands, JCAP 0812, 004 (2008), 0809.4646.

[52] C. Hikage, K. Koyama, T. Matsubara, T. Takahashi, and M. Yamaguchi, (2008), 0812.3500.

[53] M. Kawasaki, K. Nakayama, T. Sekiguchi, T. Suyama, and F. Takahashi, JCAP 0811, 019 (2008), 0808.0009.

[54] Q. G. Huang, JCAP 0811 (2008) 005 arXiv:0808.1793 [hep-th]].

[55] X. Gao and B. Hu, (2009), 0903.1920.

[56] Y.-F. Cai and H.-Y. Xia, (2009), 0904.0062.

[57] D. Langlois, S. Renaux-Petel, and D. A. Steer, (2009), 0902.2941.

[58] X. Gao, (2009), 0904.4187.

[59] Q.-G. Huang, (2009), 0903.1542.

[60] Q. G. Huang, arXiv:0904.2649 [hep-th].

[61] J. Khoury and F. Piazza, arXiv:0811.3633 [hep-th].

[62] C. Armendariz-Picon, T. Damour and V. F. Mukhanov, Phys. Lett. B 458 (1999) 209 arXiv:hep-th/9904075.

[63] J. Garriga and V. F. Mukhanov, Phys. Lett. B 458 (1999) 219 arXiv:hep-th/9904176.

[64] E. Silverstein and D. Tong, Phys. Rev. D 70 (2004) 103505 arXiv:hep-th/0310221.

[65] X. Chen, Phys. Rev. D 71 (2005) 063506 arXiv:hep-th/0408084.

[66] X. Chen, JHEP 0508 (2005) 045 arXiv:hep-th/0501184.

[67] X. Chen, Phys. Rev. D 72 (2005) 123518 arXiv:astro-ph/0507053].

[68] S. Kecskemeti, J. Maiden, G. Shiu and B. Underwood, JHEP 0609, 076 (2006) arXiv:hep-th/0605189.

[69] J. E. Lidsey and D. Seery, Phys. Rev. D75, 043505 (2007), astro-ph/0610398.

[70] D. Baumann and L. McAllister, Phys. Rev. D 75, 123508 (2007) arXiv:hep-th/0610285].

[71] R. Bean, S. E. Shandera, S. H. Henry Tye and J. Xu, JCAP 0705, 004 (2007) arXiv:hep-th/0702107].

[72] J. E. Lidsey and I. Huston, JCAP 0707, 002 (2007) arXiv:0705.0240 [hep-th]].

[73] H. V. Peiris, D. Baumann, B. Friedman, and A. Cooray, Phys. Rev. D76, 103517 (2007), 0706.1240.

[74] T. Kobayashi, S. Mukohyama and S. Kinoshita, JCAP 0801, 028 (2008) arXiv:0708.4285 [hep-th]].

[75] L. Lorenz, J. Martin, and C. Ringeval, JCAP 0804, 001 (2008), 0709.3758.

[76] R. Bean, X. Chen, H. Peiris and J. Xu, Phys. Rev. D 77, 023527 (2008) arXiv:0710.1812 [hep-th]].

[77] S. Bird, H. V. Peiris, and D. Baumann, (2009), 0905.2412.

[78] D. A. Easson, R. Gregory, D. F. Mota, G. Tasinato and I. Zavala, JCAP 0802 (2008) 010 [arXiv:0709.2666 [hep-th]].

[79] A. A. Starobinsky and J. Yokoyama, arXiv:gr-qc/9502002.

[80] M. x. Huang, G. Shiu and B. Underwood, Phys. Rev. D 77 (2008) 023511 arXiv:0709.3299 [hep-th]].

[81] D. Langlois and S. Renaux-Petel, JCAP 0804 (2008) 017 arXiv:0801.1085 [hep-th]].

[82] L. Boubekeur and D. H. Lyth, Phys. Rev. D 73, 021301 (2006) arXiv:astro-ph/0504046. 
[83] L. Alabidi and D. H. Lyth, JCAP 0605, 016 (2006) arXiv:astro-ph/0510441.

[84] N. Kogo and E. Komatsu, Phys. Rev. D 73, 083007 (2006) arXiv:astro-ph/0602099.

[85] X. Chen, B. Hu, M. x. Huang, G. Shiu and Y. Wang, arXiv:0905.3494 [astro-ph.CO].

[86] F. Arroja, S. Mizuno, K. Koyama and T. Tanaka, arXiv:0905.3641 [hep-th].

[87] D. Babich, P. Creminelli and M. Zaldarriaga, JCAP 0408, 009 (2004) arXiv:astro-ph/0405356.

[88] W. Hu, Phys. Rev. D 64, 083005 (2001) arXiv:astro-ph/0105117.

[89] T. Okamoto and W. Hu, Phys. Rev. D 66, 063008 (2002) arXiv:astro-ph/0206155.

[90] P. Creminelli, L. Senatore and M. Zaldarriaga, JCAP 0703, 019 (2007) arXiv:astro-ph/0606001.

[91] R. Arnowitt, S. Deser and C. W. Misner, Phys. Rev. 117 (1960) 1595.

[92] R. G. Leigh, Mod. Phys. Lett. A 4, 2767 (1989).

[93] C. Gordon, D. Wands, B. A. Bassett and R. Maartens, Phys. Rev. D 63 (2001) 023506 arXiv:astro-ph/0009131.

[94] S. Weinberg, Phys. Rev. D 72 (2005) 043514 arXiv:hep-th/0506236.

[95] D. Wands, N. Bartolo, S. Matarrese and A. Riotto, Phys. Rev. D 66 (2002) 043520 arXiv:astro-ph/0205253.

[96] S. Mizuno et al., in preparation. 